\title{
Impairment of the Missing Fundamental Phenomenon in Individuals with Alzheimer's Disease: A Neuropsychological and Voxel-Based Morphometric Study
}

\author{
Makiko Abe ${ }^{a}$ Ken-ichi Tabei ${ }^{a, b} \quad$ Masayuki Satoh $^{a} \quad$ Mari Fukuda $^{a}$ \\ Hironobu Daikuhara ${ }^{c}$ Mariko Shigad Hirotaka Kida ${ }^{a}$ \\ Hidekazu Tomimoto ${ }^{a}$ b \\ a Department of Dementia Prevention and Therapeutics, Graduate School of Medicine, \\ Mie University, Mie, Japan; 'b Department of Neurology, Graduate School of Medicine, \\ Mie University, Mie, Japan; ' Graduate School of Medicine, Mie University, Mie, Japan; \\ dMie Prefectural Dementia-Related Disease Medical Center, Mie, Japan
}

\section{Keywords}

Alzheimer's disease - Dementia - Pitch perception - Missing fundamental phenomenon · Magnetic resonance imaging $\cdot$ Voxel-based morphometry

\begin{abstract}
Background/Aims: The missing fundamental phenomenon (MFP) is a universal pitch perception illusion that occurs in animals and humans. In this study, we aimed to determine whether the MFP is impaired in patients with Alzheimer's disease (AD) using an auditory pitch perception experiment. We further examined anatomical correlates of the MFP in patients with $A D$ by measuring gray matter volume (GMV) on magnetic resonance images via voxel-based morphometric analysis. Methods: We prospectively enrolled 29 patients with AD and 20 healthy older adults. Auditory stimuli included 12 melodies of Japanese nursery songs that were expected to be familiar to participants. We constructed the melodies using pure and missing fundamental tones (MFTs). Results: Patients with AD exhibited significantly poorer performance on the MFT task than healthy controls. MFT scores were positively correlated with GMV in the bilateral insula and temporal poles, left inferior frontal gyrus, right entorhinal cortex, and right cerebellum. Conclusions: These results suggest that impairments in the MFP represent a manifestation of the degeneration of auditory-related brain regions in AD. Further studies are required to more fully elucidate the neural mechanisms underlying auditory impairments in patients with $A D$ and related dementia disorders.


Abe et al.: Impairment of the Missing Fundamental Phenomenon in Individuals with Alzheimer's Disease: A Neuropsychological and Voxel-Based Morphometric Study

\section{Introduction}

Speech, environmental, and musical sounds are complex acoustic signals whose processing is dependent on activity across different brain regions. Central auditory impairment is reportedly associated with mild memory dysfunction [1], and is considered a risk factor for all types of dementia [2,3]. Impaired central auditory processing is highly prevalent in individuals with Alzheimer's disease (AD) and in those with mild cognitive impairment [4]. Indeed, previous studies have reported that patients with AD respond poorly to speech sounds $[5,6]$ and exhibit decreases in the ability to parse sound sources in the auditory environment (i.e., auditory scene analysis) [7, 8]. Additionally, patients with AD exhibit grouplevel deficits in global pitch (melody contour) processing when attempting to assess musical patterns with dimensions of pitch and temporal information [9].

Most complex sounds such as speech, environmental sounds, and music consist of a fundamental tone and its overtones. Humans usually perceive the pitch as corresponding to the lowest tone (i.e., the fundamental tone) [10] (Fig. 1). However, in some cases, harmonic sounds form a missing fundamental complex that contains no spectral energy at the fundamental tone; nevertheless, they produce a pitch that matches the fundamental tone, a process referred to as the missing fundamental phenomenon (MFP). This universal pitch perception illusion occurs in animals [11-13], such as monkeys [14-16] and birds [17], as well as in 3- to 4-month-old human infants [18-24], and was suggested as a perception model [25, 26].

MFP is often used to investigate the cognitive processes underlying pitch perception. Previous studies have revealed that temporal areas of the neocortex play a role in the MFP. Zatorre [27] compared 64 patients with unilateral temporal-lobe excisions and 18 healthy controls using an MFP pitch perception task. Participants who had undergone right temporal lobectomy involving excision of Heschl's gyri committed significantly more errors than the healthy control group. These results suggest that Heschl's gyrus and the surrounding cortex in the right cerebral hemisphere play a crucial role in extracting the pitch corresponding to the fundamental tone from a complex tone. Several studies pairing brain imaging with magnetoencephalography (MEG) have reported that MFP processes occur in the auditory cortex in healthy participants [28-30], mainly at the transverse temporal and superior temporal gyri [31]. In addition, these processes were generally lateralized to the right cerebral hemisphere $[32,33]$.

Although hearing abnormalities are also observed in patients with $\mathrm{AD}[2,3]$, the processes underlying these deficits remain poorly understood. We hypothesized that hearing abnormalities due to AD-related neurodegeneration affect pitch perception; therefore, patients with AD may not experience the MFP. To test this hypothesis, we conducted an auditory pitch perception experiment to assess the MFP in patients with AD. In addition, we aimed to identify possible anatomical correlates of the MFP in patients with AD by measuring gray matter volume (GMV) via voxel-based morphometric analysis of structural magnetic resonance images (MRIs) for each participant.

\section{Materials and Methods}

\section{Participants}

Our study followed the clinical guidelines of the Ethics Committee of Mie University Hospital in Japan, was approved by the institutional review board (2520), and adhered to the tenets of the Declaration of Helsinki. Following a thorough explanation of all procedures, written informed consent was obtained from all participants or their caregivers. We prospectively enrolled 29 patients (AD group) who had consulted with the memory clinic 
Fig. 1. Missing fundamental on $\mathrm{C}$ $65(\mathrm{~Hz})$, with harmonics. The $\mathrm{Hz}$ values are listed (divisible by fundamental tone 65).
Abe et al.: Impairment of the Missing Fundamental Phenomenon in Individuals with Alzheimer's Disease: A Neuropsychological and Voxel-Based Morphometric Study

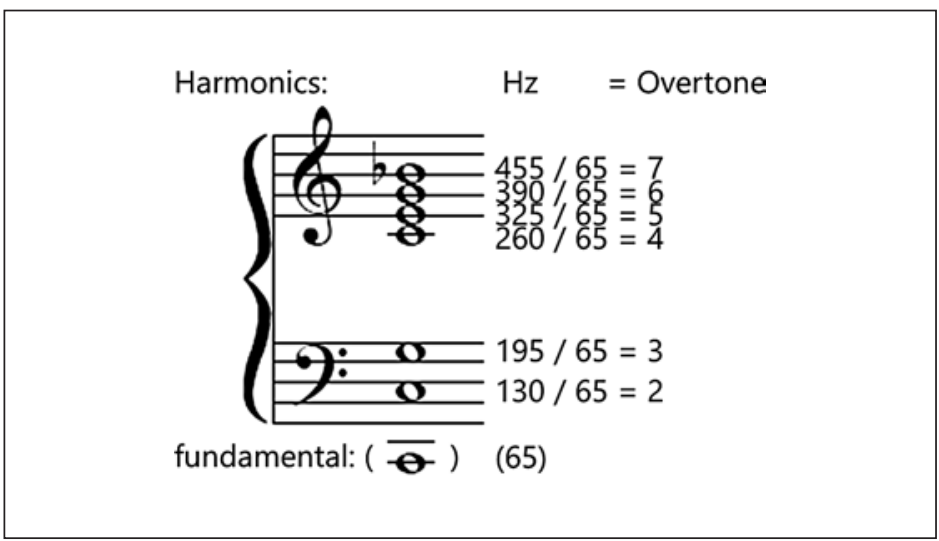

at our hospital between April 2015 and May 2016, and who fulfilled the following inclusion criteria: (1) diagnosis of probable AD based on criteria proposed by the Neurological and Communicative Disorders and Stroke-Alzheimer's Disease and Related Disorders Association (NINCDS-ADRDA) [34], (2) hearing ability and eyesight that did not interfere with activities of daily living (ADLs) or completion of neuropsychological assessments, (3) an Ascertain Dementia 8 (AD8) [35] score of 2 points or more, and (4) completion of neuropsychological and MRI assessments. Patients were excluded if they (1) declined neuropsychological and MRI assessments, (2) were not diagnosed with AD, or (3) exhibited normal cognitive function.

We also recruited 20 healthy older adults (control group) from among the caregivers of patients with AD visiting our hospital during the same period. Inclusion criteria for healthy controls were as follows: (1) capable of independently completing ADLs; (2) absence of neurological symptoms, including progressive disturbance of memory, for the preceding several years; (3) absence of neuropsychological or psychiatric symptoms; (4) an AD8 [35] score of 1 point or less; and (5) hearing ability and eyesight that did not interfere with ADLs or completion of neuropsychological assessments.

\section{Behavioral Experiment}

For patients with $\mathrm{AD}$, intellectual function was assessed using the Mini-Mental State Examination (MMSE) [36]. An assessment of constructional ability was conducted based on the method described by Strub and Black [37]. Participants were shown and then asked to draw a cube. Each drawing was assigned a score from 0 to 3 (0: poor; 1: fair; 2: good; or 3: excellent).

The auditory stimuli included 12 melodies of Japanese nursery songs expected to be familiar to Japanese older adults. We constructed the melodies using pure tones (PT) and missing fundamental tones (MFT) in MATLAB R2012a (MathWorks, Natick, MA, USA) (Fig. 2). The participants were presented with 12 PT melodies in the PT task and 12 MFT melodies in the MFT task. The tasks were presented in a random order. In these tasks, participants were asked what melody they heard and replied by either pointing to the figure of the correct song, singing the song, naming the title of the song, or reporting it as an unknown song or unrecognizable melody. Accuracy rates for the PT and MFT tasks were regarded as the PT and MFT scores, respectively. When participants responded incorrectly, we were unable to determine whether they did not know the melody or whether MFP did not occur. Therefore, we also calculated the accuracy rate for the MFT task using only the melodies with correct responses in the PT task to derive the MFT/PT score. 
Fig. 2. Example of an auditory stimulus in the form of the melody of a Japanese nursery song (Momo-taro), which was expected to be familiar to Japanese older adults. The melody was constructed using pure tones (a) and missing fundamental tones (b). FT, fundamental tone.

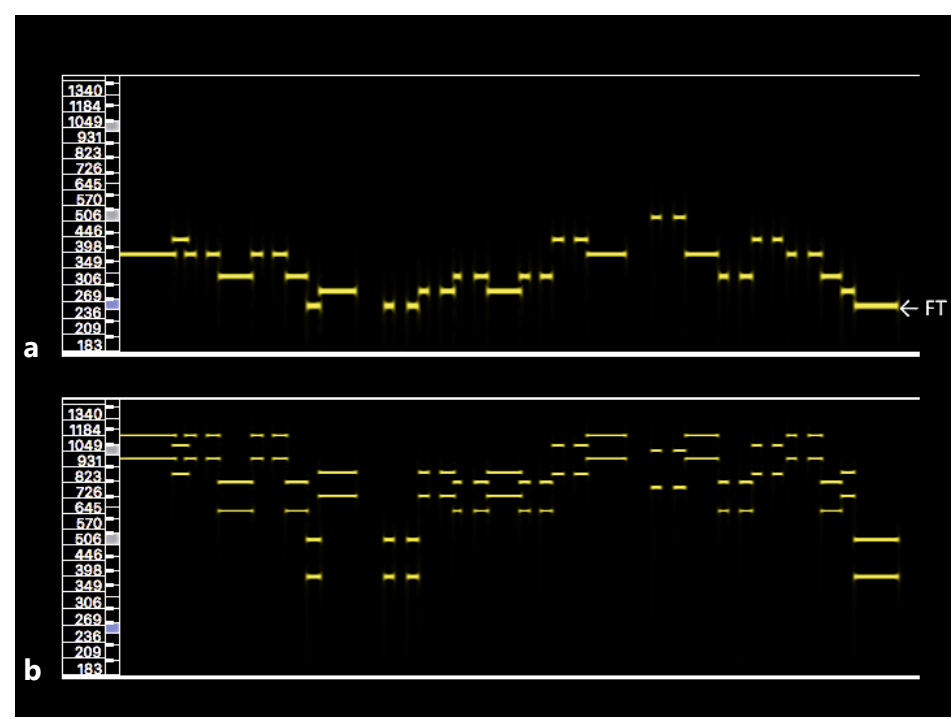

\section{MR Image Analysis}

T1-weighted MR images were obtained using a 3T MRI scanner (Achieva or Ingenia, Philips Medical System, Amsterdam, The Netherlands). The parameters for T1-weighted images were as follows: repetition time, $7.6 \mathrm{~ms}$; echo time, $3.6 \mathrm{~ms}$; flip angle, $8^{\circ}$; field of view, $250 \times 250 \mathrm{~mm}$; in-plane resolution, $1.04 \times 1.04 \mathrm{~mm}$; and slice thickness, $0.7 \mathrm{~mm}$.

MRI data were analyzed using SPM12 (Wellcome Trust Centre for Neuroimaging, University College London, UK) running on MATLAB R2012a (MathWorks). During preprocessing, images were realigned to the anterior-posterior commissure line using an automated MATLAB script. Subsequently, the images were visually inspected to locate possible scan issues, such as field distortion and movement artifacts. Reoriented images were corrected for intensity inhomogeneity and segmented into GM, white matter (WM), cerebrospinal fluid, and other tissues outside the brain using SPM12 tissue probability maps. The images were registered in the East Asian brains International Consortium for Brain Mapping template using affine regularization. We created a population-specific template using the SPM12 DARTEL template procedure to investigate the relationship between MFT/PT score and GM at the whole-brain level in the AD group. The GM and WM segments were input into high-dimensional DARTEL to create nonlinear modulated-normalized GM and WM images, which were smoothed using a Gaussian kernel of 8-mm full width at half maximum. For whole-brain and multiple regression analyses, we assessed statistical significance at a voxel cluster threshold of $p<0.001$ (uncorrected); contiguous clusters of at least 10 voxels were reported.

\section{Data Analysis}

Statistical analyses were conducted using independent $t$ tests for continuous variables, $\chi^{2}$ tests for dichotomous variables, and Mann-Whitney U tests for nonparametric data. Correlation coefficients were interpreted as weak $(0.00-0.25)$, fair $(0.25-0.50)$, moderate $(0.50-$ $0.75)$, and strong (0.75-1.0) associations. For all statistical analyses, differences with a $p$ value of $<0.05$ were deemed statistically significant. All analyses were performed using IBM SPSS Statistics version 22.0 (Armonk, NY, USA). 


\begin{tabular}{l|l|}
\hline DOI: 10.1159/000486331 & $\begin{array}{l}\text { C } 2018 \text { The Author(s). Published by S. Karger AG, Basel } \\
\text { www.karger.com/dee }\end{array}$ \\
\hline
\end{tabular}

Abe et al.: Impairment of the Missing Fundamental Phenomenon in Individuals with Alzheimer's Disease: A Neuropsychological and Voxel-Based Morphometric Study

Table 1. Clinical characteristics and neuropsychological assessment results for all participants

\begin{tabular}{lccr}
\hline & AD group & Control group & $p$ value \\
\hline Participants & $29(13 \mathrm{men})$ & $20(10 \mathrm{men})$ & 0.721 \\
Age, years & $74.79 \pm 8.59$ & $73.00 \pm 10.84$ & 0.768 \\
AD8 & $3.38 \pm 1.18$ & $0.10 \pm 0.31$ & $<0.001$ \\
\hline
\end{tabular}

Data are presented as $n$ or mean \pm SD. AD, Alzheimer's disease.

Table 2. Comparisons of PT, MFT, and MFT/PT scores between the $\mathrm{AD}$ and control groups

\begin{tabular}{llll}
\hline & AD group & Control group & $p$ value \\
\hline PT score & $0.97 \pm 0.11$ & $0.99 \pm 0.04$ & 0.333 \\
MFT score & $0.95 \pm 0.07$ & $0.98 \pm 0.04$ & 0.118 \\
MFT/PT score & $0.86 \pm 0.18$ & $0.97 \pm 0.08$ & 0.007 \\
\hline
\end{tabular}

Data are presented as mean $\pm \mathrm{SD}$. AD, Alzheimer's disease; MFT, missing fundamental tone; PT, pure tone.

Table 3. Correlation results for the analysis of associations between task scores and patient characteristics/ clinical scores

\begin{tabular}{|c|c|c|c|c|c|c|}
\hline & Age, years & Sex & $\begin{array}{l}\text { Education, } \\
\text { years }\end{array}$ & $\begin{array}{l}\text { Dementia } \\
\text { duration, years }\end{array}$ & MMSE score & $\begin{array}{l}\text { Constructional } \\
\text { ability score }\end{array}$ \\
\hline \multicolumn{7}{|l|}{ MFT } \\
\hline$r$ & -0.007 & -0.049 & -0.301 & 0.027 & 0.141 & -0.212 \\
\hline$p$ & 0.973 & 0.801 & 0.112 & 0.891 & 0.465 & 0.269 \\
\hline \multicolumn{7}{|l|}{ PT } \\
\hline$r$ & -0.110 & -0.249 & -0.024 & 0.112 & 0.014 & 0.028 \\
\hline$p$ & 0.569 & 0.193 & 0.904 & 0.564 & 0.941 & 0.885 \\
\hline \multicolumn{7}{|c|}{ MFT/PT } \\
\hline$r$ & 0.107 & 0.073 & -0.068 & 0.310 & 0.209 & -0.110 \\
\hline$p$ & 0.581 & 0.705 & 0.728 & 0.102 & 0.276 & 0.570 \\
\hline
\end{tabular}

MFT, missing fundamental tone; PT, pure tone; MMSE, Mini-Mental State Examination.

\section{Results}

\section{Demographic Data}

Table 1 shows the clinical characteristics and neuropsychological assessment results of the participants. There were no significant differences between the AD and control groups with respect to the mean age and sex ratio ( $p=0.768$ and $p=0.721$, respectively). However, there was a significant difference between the AD and control groups with respect to the AD8 ratio $(p<0.001)$.

\section{Behavioral Data}

Table 2 shows the results of the comparisons between the AD and control groups. There were no significant differences in the accuracy rate between patients and healthy controls for either PT or MFT scores ( $p=0.333$ and $p=0.118$, respectively). Additionally, we calculated the MFT/PT score using only those melodies for which participants provided correct answers 


\begin{tabular}{l|l}
\hline DOI: 10.1159/000486331 & $\begin{array}{l}\text { (c) } 2018 \text { The Author(s). Published by S. Karger AG, Basel } \\
\text { www.karger.com/dee }\end{array}$ \\
\hline
\end{tabular}

Abe et al.: Impairment of the Missing Fundamental Phenomenon in Individuals with Alzheimer's Disease: A Neuropsychological and Voxel-Based Morphometric Study

Table 4. Demographic characteristics of participants included in the VBM analysis

\begin{tabular}{lc}
\hline Variable & VBM AD group \\
\hline Participants & $12(4$ men) \\
Age, years & $72.42 \pm 11.31$ \\
Education, years & $10.29 \pm 2.06$ \\
Dementia duration, years & $1.73 \pm 1.74$ \\
MMSE score & $22.58 \pm 3.88$ \\
Constructional ability score & $1.67 \pm 0.47$ \\
MFT/PT score & $0.82 \pm 0.23$ \\
\hline
\end{tabular}

Values are presented as $n$ or mean \pm SD. AD, Alzheimer's disease; $\mathrm{MFT} / \mathrm{PT}$, missing fundamental tone/pure tone; MMSE, Mini-Mental State Examination; VBM, voxel-based morphometry.

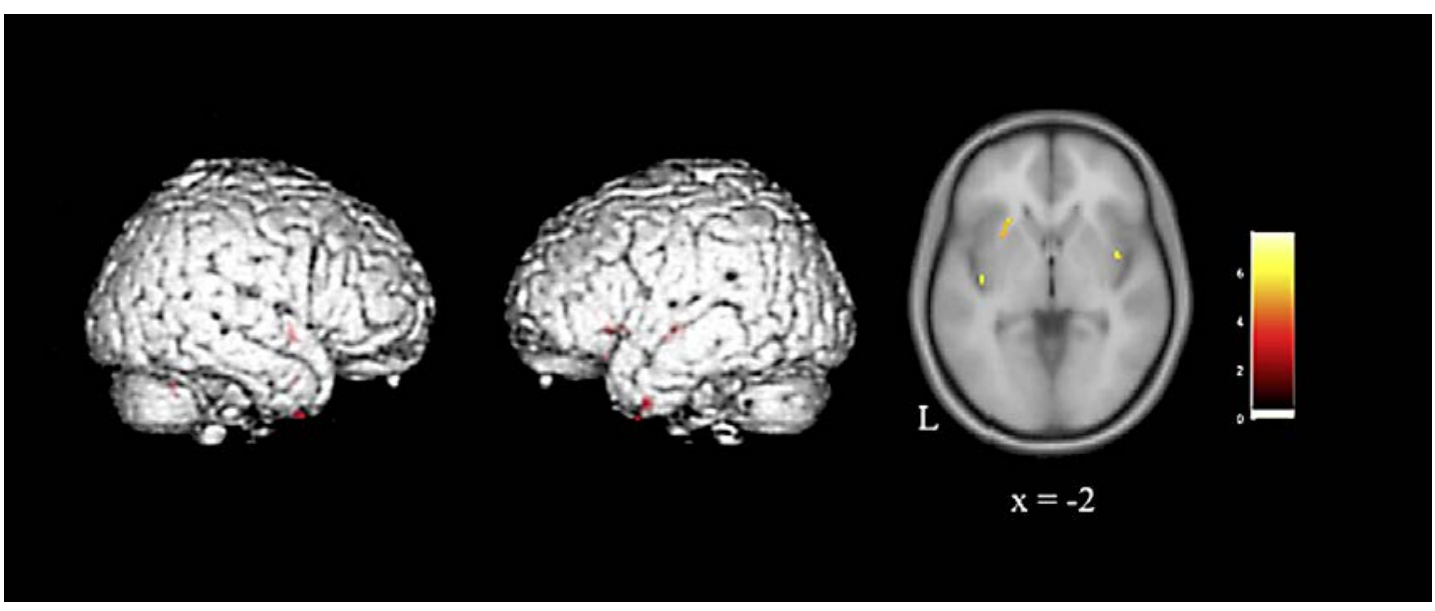

Fig. 3. Magnetic resonance image showing regions in which the accuracy rate for the missing fundamental tone/pure tone was significantly correlated with gray matter volume. L, left.

in the PT task (see the section Behavioral Experiment). The comparison of the MFT/PT scores revealed that the $\mathrm{AD}$ group performed significantly worse than the control group $(p=0.007$; Table 2).

Table 3 shows the correlation results for associations between MFT, PT, and MFT/PT scores and patient characteristics/neuropsychological assessment results. No significant correlations were observed for any combination. The mean level of education in the AD group was $11.28 \pm 2.58$ years, while the mean symptom duration was $2.58 \pm 1.68$ years. The mean MMSE score for patients with AD was $22.79 \pm 4.38$, while the mean constructional ability score was $1.79 \pm 0.57$ (Table 3 ).

\section{MRI Data}

We performed voxel-based morphometric analysis of MRI data from 12 patients with AD who had undergone brain MRI within 3 months of the behavioral study. Table 4 shows the clinical characteristics, neuropsychological assessment results, and MFP task results for these 12 patients. There were significant correlations between the MFT/PT accuracy rate and GMV in several regions. MFT/PT scores were positively correlated with GMV in the left and right insula, left and right temporal pole, left inferior frontal gyrus, right entorhinal cortex, and right cerebellum ( $p<0.001$, uncorrected; Fig. 3; Table 5). 
Abe et al.: Impairment of the Missing Fundamental Phenomenon in Individuals with Alzheimer's Disease: A Neuropsychological and Voxel-Based Morphometric Study

Table 5. Cluster sizes, peak locations, and statistical values for each contrast

\begin{tabular}{|c|c|c|c|c|c|c|c|}
\hline \multirow[t]{2}{*}{$\mathrm{L} / \mathrm{R}$} & \multirow[t]{2}{*}{ Area } & \multirow[t]{2}{*}{ BA } & \multicolumn{3}{|c|}{ Talairach coordinates, $\mathrm{mm}$} & \multirow[t]{2}{*}{$Z$ value } & \multirow{2}{*}{$\begin{array}{l}\text { Cluster size } \\
\text { in voxels }\end{array}$} \\
\hline & & & $x$ & $y$ & $z$ & & \\
\hline $\mathrm{L}$ & Insula & 13 & -39 & -16 & -1 & 4.01 & 58 \\
\hline $\mathrm{R}$ & Insula & 13 & 36 & 3 & -7 & 3.99 & 60 \\
\hline $\mathrm{R}$ & Insula & 13 & 40 & 0 & -2 & 3.56 & \\
\hline $\mathrm{R}$ & Temporal pole & 38 & 34 & 4 & -41 & 3.71 & 58 \\
\hline $\mathrm{L}$ & Insula & 13 & -26 & 20 & -3 & 3.43 & 58 \\
\hline $\mathrm{L}$ & Insula & 13 & -30 & 11 & -3 & 3.25 & \\
\hline $\mathrm{L}$ & Inferior frontal gyrus & 47 & -20 & 21 & -14 & 3.41 & 11 \\
\hline $\mathrm{L}$ & Temporal pole & 38 & -38 & 0 & -33 & 3.39 & 59 \\
\hline $\mathrm{R}$ & Entorhinal & 28 & 21 & 5 & -23 & 3.37 & 24 \\
\hline $\mathrm{R}$ & Cerebellum & & 33 & -58 & -27 & 3.25 & 30 \\
\hline
\end{tabular}

BA, Brodmann area; L, left; R, right.

\section{Discussion}

In the present study, we conducted an auditory pitch-perception experiment based on the MFT to investigate whether auditory abnormalities can be detected in patients with AD. Our findings can be summarized as follows: (1) There were no significant differences in accuracy rates between patients with AD and healthy controls for the PT task; (2) patients of the AD group exhibited significantly poorer MFT/PT scores than healthy controls; (3) there were no significant correlations between PT or MFT/PT scores and patient characteristics or neuropsychological assessment results; (4) MFT/PT scores were positively correlated with the volumes of the left and right insula, left and right temporal pole, left inferior frontal gyrus, right entorhinal cortex, and cerebellum. To our knowledge, the present study is the first to suggest that the MFP is attenuated in patients with AD.

We observed significant differences in MFT/PT scores between the patient and healthy control groups, although there was no significant difference in the number of correct answers in the PT task. We then compared MFT/PT scores between the AD and control groups using data for only those melodies that had been correctly identified in the PT task. If MFP had occurred, participants would have been able to recognize the melody as similar to that in the PT task. However, comparisons of MFT/PT scores revealed that the AD group performed worse than the control group. This result indicates that the AD group could not perceive the melody in the MFT task, supporting our hypothesis that patients with AD exhibit impairments with regard to the MFP. Moreover, these results suggest that functional deterioration of auditory perception due to AD occurs at primitive levels of hearing processing. However, because we did not observe a significant correlation with MMSE or other neuropsychological scores, it is unlikely that the attenuation of the MFP in patients with AD was caused by cognitive impairment. Our results are consistent with previous research, which has shown that hearing impairments can be observed beginning in the early stages of AD [4], and that cognitive function and hearing impairment are not correlated [38]. Moreover, Idrizbegovic et al. [4] reported no significant differences in the performance of patients with AD and healthy controls on PT tasks, in accordance with the results of the present study.

We further observed that GMV in several brain regions was correlated with MFT/PT scores in the AD group, including the bilateral insula, bilateral temporal pole, left inferior frontal gyrus, right entorhinal cortex, and right cerebellum. Of note was the correlation between MFT/PT scores and the GMV of the temporal pole, as this region has been highly 
implicated in tone- and melody-related auditory processing. Patterson et al. [39] reported that the temporal pole is involved in melody recognition. Satoh et al. [40] reported a case of amusia in a patient with infarction in the anterior portion of the bilateral temporal lobes, who exhibited impairments in recognizing familiar melodies and chords. Satoh et al. [41] also investigated the role of the anterior portion of the temporal lobes in music perception in nonmusicians using positron emission tomography. The authors reported that the anterior portion of the bilateral temporal lobes is vital for the discrimination of chords, and suggested that the temporal pole is involved in the organization of melody and chord perception. Therefore, we postulate that the temporal pole participates in the neural processing of multiple simultaneous tones as a whole, which is related to the occurrence of MFP. Additionally, previous results have suggested that the inferior frontal gyrus is involved in the binding of lyrics and melodies [42], recognition of familiar melodies [43], and the perception of melodies [44]. Therefore, the observed association between the MFP and GMV in the temporal pole and inferior frontal gyrus may reflect the recognition of common nursery song melodies. In contrast, previous studies have shown an association between MFTs and the superior temporal gyrus (STG) [28-33, 45], although this region was not significantly correlated with MFTs in our study. This may be because the STG, which includes the primary auditory cortex, rarely becomes atrophic in patients with AD.

Furthermore, we detected correlations between MFT/PT scores and GMV in the left and right insula. The insula may be engaged in processing aspects of auditory motion or integrating spatial information with other sound characteristics [46-48]. While this region has not been implicated in the MFP in previous MEG studies, this may be because MEG cannot penetrate deep brain structures. Our results indicate that the insula may be involved in the MFP, and that the failure to detect such involvement in previous studies may have been due to technical factors.

The following limitations should be considered when interpreting our results. First, in conventional research, a single tone is often used as the stimulus. However, nursery song melodies were used in the current study to make the task easier for patients with AD. Cognitive processing may be more complex in this scenario than that associated with single tones. Additionally, future studies should include control conditions in which participants are presented with unfamiliar melodies, in order to determine whether all participants had deduced that the presented melodies were intended to be familiar. Second, we were unable to conduct neuropsychological examinations and MRI scans of healthy controls. Consequently, it was not possible to compare MRI results between the groups. Third, only a small proportion of included patients had available MRI data. Finally, we were unable to investigate forms of dementia other than AD. Future studies should assess the mechanisms underlying the development of MFP in other types of dementia (e.g., dementia with Lewy bodies and vascular dementia) and compare the results with those for AD.

In conclusion, we observed impaired MFP task performance in patients with AD when compared to that of healthy controls. Performance was associated with GMV reductions in several frontal, temporal, and subcortical regions. These results suggest that MFP performance is a manifestation of the degeneration of auditory-related brain regions in AD. Further studies are required to more fully elucidate the neural mechanisms underlying auditory impairments in patients with $\mathrm{AD}$ and related dementia disorders.

\section{Acknowledgments}

We thank Dr. Hideo Eda for his support of the MF stimuli program. 
Abe et al.: Impairment of the Missing Fundamental Phenomenon in Individuals with Alzheimer's Disease: A Neuropsychological and Voxel-Based Morphometric Study

\section{Disclosure Statement}

The authors have no conflicts of interest to report.

\section{Funding Sources}

This work was supported by the Japan Society for the Promotion of Science Grants-in-Aid for Young Scientists (B) 25870325 and 17K17811 and Scientific Research (C) 15KT0095 to K.T.

\section{Author Contributions}

Conception and design of the experiments: M.S., K.T. Performance of experiments: M.A., M.F., H.D., and M.Sh. Data analysis: M.A. and K.T. Writing of the paper: M.A., K.T., and M.S. Analysis and interpretation of the data: H.K. Supervision and interpretation of the data: H.T.

\section{References}

1 Gates GA, Anderson ML, Feeney MP, McCurry SM, Larson EB: Central auditory dysfunction in older persons with memory impairment or Alzheimer dementia. Arch Otolaryngol Head Neck Surg 2008;134:771-777.

2 Lin FR, Metter EJ, O’Brien, RJ, Resnick SM, Zonderman AB, Ferrucci L: Hearing loss and incident dementia. Arch Neurol 2011;68:214-220.

3 Hung S-CC, Liao K-FF, Muo C-HH, Lai SW, Chang CW, Hung HC: Hearing loss is associated with risk of Alzheimer's disease: a case-control study in older people. J Epidemiol 2015;25:517-521.

4 Idrizbegovic E, Hederstierna C, Dahlquist M, Kämpfe Nordström C, Jelic V, Rosenhall U: Central auditory function in early Alzheimer's disease and in mild cognitive impairment. Age Ageing 2011;40:249-254.

5 Gates GA, Besier A, Rees TS, D’Agostino RB, Wolf PA: Central auditory dysfunction may precede the onset of clinical dementia in people with probable Alzheimer's disease. J Am Geriatr Soc 2002;50:482-488.

6 Ouchi Y, Meguro K, Akanuma K, Kato Y, Yamaguchi S: Normal hearing ability but impaired auditory selective attention associated with prediction of response to donepezil in patients with Alzheimer's disease. Behav Neurol 2015:540348.

7 Goll JC, Kim LG, Ridgway GR, Hailstone JC, Lehmann M, Buckley AH, Crutch SJ, Warren JD: Impairments of auditory scene analysis in Alzheimer's disease. Brain 2012;135:190-200.

8 Golden HL, Nicholas JM, Yong KX, Downey LE, Schott JM, Mummery CJ, Crutch SJ, Warren JD: Auditory spatial processing in Alzheimer's disease. Brain 2015;138:189-202.

9 Golden HL, Clark CN, Nicholas JM, Cohen MH, Slattery CF, Paterson RW, Foulkes AJM, Schott JM, Mummery CJ, Crutch SJ, Warren JD: Music perception in dementia. J Alzheimers Dis 2017;55:933-949.

10 Zatorre RJ: Neuroscience: finding the missing fundamental. Nature 2005;436:1093-1094.

11 Henry KR: Sharply tuned cochlear nerve ensemble periodicity responses to sonic and ultrasonic frequencies. J Comp Physiol A 1997;181:239-246.

12 Qin L, Sakai M, Chimoto S, Sato Y: Interaction of excitatory and inhibitory frequency-receptive fields in determining fundamental frequency sensitivity of primary auditory cortex neurons in awake cats. Cereb Cortex 2015;15:1371-1383.

13 Shofner WP: Perception of the missing fundamental by chinchillas in the presence of low-pass masking noise. J Assoc Res Otolaryngol 2011;12:101-112.

14 Tomlinson RW, Schwarz DW: Perception of the missing fundamental in nonhuman primates. J Acoust Soc Am 1988;84:560-565.

15 Fishman YI, Reser DH, Arezzo JC, Steinschneider M: Pitch vs spectral encoding of harmonic complex tones in primary auditory cortex of the awake monkey. Brain Res 1998;786:18-30.

16 Bendor D, Wang X: The neuronal representation of pitch in primate auditory cortex. Nature 2005;436:11611165.

17 Cynx J, Shapiro M: Perception of missing fundamental by a species of songbird (Sturnus vulgaris). J Comp Psychol 1986;100:356-360.

18 Clarkson MG, Clifton RK: Infant pitch perception: evidence for responding to pitch categories and the missing fundamental. J Acoust Soc Am 1985; 77:1521-1528. 


\begin{tabular}{l|l}
\hline DOI: 10.1159/000486331 & (c) 2018 The Author(s). Published by S. Karger AG, Basel \\
\hline
\end{tabular}

19 Clarkson MG, Clifton RK: Infants' pitch perception: inharmonic tonal complexes. J Acoust Soc Am 1995;98: 1372-1379.

20 Clarkson MG, Rogers EC: Infants require low-frequency energy to hear the pitch of the missing fundamental. J Acoust Soc Am 1995; 98:148-154.

21 Montgomery CR, Clarkson MG: Infants' pitch perception: masking by low- and high-frequency noises. J Acoust Soc Am 1997; 102:3665-3672.

22 He C, Trainor LJ: Finding the pitch of the missing fundamental in infants. J Neurosci 2009;29:7718-8822.

23 Lau BK, Werner LA: Perception of missing fundamental pitch by 3- and 4-month-old human infants, J Acoust Soc Am 2012;132:3874-3882.

24 Lau BK, Werner LA: Perception of the pitch of unresolved harmonics by 3- and 7-month-old human infants. J Acoust Soc Am 2014;136:760-767.

25 Kerr RR, Burkitt AN, Thomas DA, Gilson M, Grayden DB: Delay selection by spike-timing-dependent plasticity in recurrent networks of spiking neurons receiving oscillatory inputs. PLoS Comput Biol 2013;9:e1002897.

26 Barzelay 0, Furst M, Barak 0: A new approach to model pitch perception using sparse coding. PLoS Comput Biol 2017;13:e1005338.

27 Zatorre RJ: Pitch perception of complex tones and human temporal-lobe function. J Acoust Soc Am 1988;84: 566-572.

28 Pantev C, Hoke M, Lütkenhöner B, Lehnertz K: Tonotopic organization of the auditory cortex: pitch versus frequency representation. Science 1989;246:486-488.

29 Schulte M, Knief A, Seither-Preisler A, Pantev C: Different modes of pitch perception and learning-induced neuronal plasticity of the human auditory cortex. Neural Plast 2002;9:161-175.

30 Monahan PJ, Souza K de, Idsardi WJ: Neuromagnetic evidence for early auditory restoration of fundamental pitch. PLoS One 2008;3:e2900.

31 Matsuwaki Y, Nakajima T, Ookushi T, Iimura J, Kunou K, Nakagawa M, Shintani M, Moriyama H, Ishikawa T: Evaluation of missing fundamental phenomenon in the human auditory cortex. Auris Nasus Larynx 2004;31: 208-211.

32 Laguitton V, Demany L, Semal C, Liégeois-Chauvel C: Pitch perception: a difference between right- and lefthanded listeners. Neuropsychologia 1998;36:201-207.

33 Fujioka T, Ross B, Okamato H, Takeshima Y, Kakigi R, Pantev C: Tonotopic representation of missing fundamental complex sounds in the human auditory cortex. Eur J Neurosci 2003;18:432-440.

34 McKhann G, Drachman D, Folstein M, Katzman R, Price D, Stadlan EM: Clinical diagnosis of Alzheimer's disease: report of the NINCDS-ADRDA Work Group under the auspices of Department of Health and Human Services Task Force on Alzheimer's Disease. Neurology 1984;34:939-944.

35 Meguro K, Kasai M, Nakamura K: Reliability and validity of the Japanese version of the AD8. Nippon Ronen Igakkai Zasshi 2015;52:61-70.

36 Folstein MF, Folstein SE, McHugh PR: "Mini-mental state." A practical method for grading the cognitive state of patients for the clinician. J Psychiatr Res 1975;12:189-198.

37 Strub RL, Black FW: The Mental Status Examination in Neurology, ed 4. Philadelphia, FA DAVIS Company, 2000, pp 93-115.

38 Gussekloo J, de Craen AJ, Oduber C, van Boxtel MP, Westendorp RG: Sensory impairment and cognitive functioning in oldest-old subjects: the Leiden 85+ Study. Am J Geriatr Psychiatry 2005;13:781-786.

39 Patterson RD, Uppenkamp S, Johnsrude IS, Griffiths TD: The processing of temporal pitch and melody information in auditory cortex. Neuron 2002;36:767-776.

40 Satoh M, Takeda K, Murakami Y, Onouchi K, Inoue K, Kuzuhara S: A case of amusia caused by the infarction of anterior portion of bilateral temporal lobes. Cortex 2005;41:77-83.

41 Satoh M, Takeda K, Nagata K, Hatazawa J, Kuzuhara S: The anterior portion of the bilateral temporal lobes participates in music perception: a positron emission tomography study. Am J Neuroradiol 2003;24:18431848.

42 Alonso I, Davachi L, Valabrègue R, Lambrecq V, Dupont S, Samson S: Neural correlates of binding lyrics and melodies for the encoding of new songs. Neuroimage 2016;127:333-345.

43 Sikka R, Cuddy LL, Johnsrude IS, Vanstone AD: An fMRI comparison of neural activity associated with recognition of familiar melodies in younger and older adults. Front Neurosci 2015;9:356.

44 Herholz SC, Halpern AR, Zatorre RJ: Neuronal correlates of perception, imagery, and memory for familiar tunes. J Cogn Neurosci 2012;24:1382-1397.

45 Pantev C, Hoke M, Lütkenhöner B, Lehnertz K: Neuromagnetic evidence of functional organization of the auditory cortex in humans. Acta Otolaryngol Suppl 1991;491:106-114; discussion 115.

46 Griffiths TD, Bench CJ, Frackowiak RSJ: Human cortical areas selectively activated by apparent sound movement. Curr Biol 1994;4:892-895.

47 Lewis JW, Beauchamp MS, DeYoe EA: A comparison of visual and auditory motion processing in human cerebral cortex. Cereb Cortex 2000;10:873-888.

48 Altmann CF, Henning M, Döring MK, Kaiser J: Effects of feature-selective attention on auditory pattern and location processing. Neuroimage 2008;41:69-79. 